REVIEW ARTICLE

\title{
Current progress of rehabilitative strategies in stem cell therapy for spinal cord injury: a review
}

\author{
Syoichi Tashiro $\mathbb{1}^{1,2 \otimes}$, Osahiko Tsuji $\mathbb{1}^{3}$, Munehisa Shinozaki $\mathbb{C}^{4}$, Takahiro Shibata ${ }^{3}$, Takashi Yoshida ${ }^{1}$, Yohei Tomioka ${ }^{5}$, Kei Unai ${ }^{1}$, \\ Takahiro Kondo ${ }^{4}$, Go Itakura ${ }^{3}$, Yoshiomi Kobayashi, ${ }^{3,6}$, Akimasa Yasuda ${ }^{3,7}$, Satoshi Nori ${ }^{3}$, Kanehiro Fujiyoshi ${ }^{3,6}$, Narihito Nagoshi ${ }^{3}$, \\ Michiyuki Kawakami ${ }^{1}$, Osamu Uemura $\mathbb{D}^{1,5}$, Shin Yamada ${ }^{2}$, Tetsuya Tsuji ${ }^{1}$, Hideyuki Okano ${ }^{4}$ and Masaya Nakamura ${ }^{3}$
}

Stem cell-based regenerative therapy has opened an avenue for functional recovery of patients with spinal cord injury (SCI). Regenerative rehabilitation is attracting wide attention owing to its synergistic effects, feasibility, non-invasiveness, and diverse and systemic properties. In this review article, we summarize the features of rehabilitation, describe the mechanism of combinatorial treatment, and discuss regenerative rehabilitation in the context of SCl. Although conventional rehabilitative methods have commonly been implemented alone, especially in studies of acute-to-subacute $\mathrm{SCl}$, the combinatorial effects of intensive and advanced methods, including various neurorehabilitative approaches, have also been reported. Separating the concept of combined rehabilitation from regenerative rehabilitation, we suggest that the main roles of regenerative rehabilitation can be categorized as conditioning/reconditioning, functional training, and physical exercise, all of which are indispensable for enhancing functional recovery achieved using stem cell therapies.

npj Regenerative Medicine (2021)6:81; https://doi.org/10.1038/s41536-021-00191-7

\section{INTRODUCTION}

Spinal cord injury $(\mathrm{SCl})$ results in various neurological sequelae in the motor, sensory, and autonomic systems. There is no treatment in the strict sense, only approaches to reduce secondary damage acute $\mathrm{SCl}$, including surgical procedures to restabilize and decompress the spinal cord and to augment blood pressure ${ }^{1}$. Rehabilitative therapies are performed after these procedures, but the injured spinal cord exhibits only a small degree of plasticity and functional recovery ${ }^{2}$. For more than two decades, stem cellbased regenerative therapy has been investigated as a state-ofthe-art treatment that is expected to change the prognosis after $\mathrm{SCl}$. Both cellular graft sources, including olfactory ensheathing cells (OECs), mesenchymal stem cells (MSCs), and neural stem/ progenitor cells (NS/PCs), and tissue graft sources, including peripheral nerve and olfactory mucosa, have been investigated. Although some of these methods have proceeded to the clinical trial stage, the transplanted stem cells do not always work as we expected and thus the current data only show limited functional recovery. One factor that markedly affects the therapeutic potential is the microenvironment, which changes over time after injury $^{3}$. Although many researchers reported that stem cell therapies have significant effects in the early phases, they lose their therapeutic potential in the chronic phase as neuronal plasticity decreases ${ }^{1,4-6}$. There are 50 -fold more patients in the chronic phase than in the acute-to-subacute phase; therefore, it is crucial to establish strategies that can be used to treat the chronically injured spinal cord ${ }^{7}$.

Recently, a strategy to combine optimal rehabilitation with regenerative treatments, called regenerative rehabilitation ${ }^{8,9}$, has been proposed based upon preclinical $^{10-12}$ and clinical ${ }^{13-15}$ research that reported a variety of mechanisms and effects on physical functions including muscle strength, gait, and the ability to perform activities of daily living $(A D L)^{9,16,17}$. This concept is concisely defined as "The application of rehabilitation protocols and principles together with regenerative medicine therapeutics toward the goal of optimizing functional recovery through tissue regeneration, remodeling, or repair" ${ }^{\prime \prime}$. Rehabilitation is suggested to promote functional integration of the graft and host neuronal system when combined with stem cell therapies ${ }^{2}$. However, to the best of our knowledge, regenerative rehabilitation following $\mathrm{SCl}$ has not been structurally summarized because research in this area is in its infancy. Therefore, this review provides an overview of the mechanisms of rehabilitation performed in combination with stem cell therapies and describes the concepts of clinical regenerative rehabilitation. In addition, we briefly introduce rehabilitative strategies more generally, independent of their combinatorial use with regenerative therapies, to provide a more integrated discussion of this area. Although regenerative medicine encompasses various treatments including cell, tissue, and organ replacement, cytokine therapy, and neurorehabilitative approaches to induce regeneration of impaired body parts and/ or systems ${ }^{18,19}$, this review specifically deals with stem cell transplantation therapies. Rehabilitation includes physical treatments utilizing electrical stimulation, magnetic stimulation, and infrared and ultrasound treatments. Here, we focus on exercise training and physical therapies used in combination with exercise.

We searched studies in the Web of Science (BIOSIS), Medline (via PubMed), Scopus, and ProQuest databases from the beginning of 1981 to 1 July 2021. Keywords related to "spinal cord injury" and "transplantation" in combination with terms related to "rehabilitation" and "training" were used in searches for preclinical research. In addition, although some functional assessments of

\footnotetext{
${ }^{1}$ Department of Rehabilitation Medicine, Keio University School of Medicine, Shinjuku, Tokyo, Japan. ${ }^{2}$ Department of Rehabilitation Medicine, Kyorin University School of Medicine, Mitaka, Tokyo, Japan. ${ }^{3}$ Department of Orthopaedic Surgery, Keio University School of Medicine, Shinjuku, Tokyo, Japan. ${ }^{4}$ Department of Physiology, Keio University School of Medicine, Shinjuku, Tokyo, Japan. ${ }^{5}$ Department of Rehabilitation, Murayama Medical Center, Musashi-Murayama, Tokyo, Japan. ${ }^{6}$ Department of Orthopaedic Surgery, Murayama Medical Center, Musashi-Murayama, Tokyo, Japan. ${ }^{7}$ Department of Orthopaedic surgery, National Defense Medical College, Tokorozawa, Saitama, Japan. email: s-tashiro@keio.jp
} 
forelimbs require training, this training is often not described in the abstract. Therefore, we closely checked the methods sections of the studies chosen using keywords related to "forelimb" instead of "rehabilitation" to determine whether rehabilitative training was implemented. Studies in which the post-therapeutic training frequency was no less than three times per week were assembled into a table. Keywords related to "spinal cord injury" and "transplantation" limited to clinical research were applied to searches for clinical studies.

\section{SCI REHABILITATION MODELS IN PRECLINICAL STUDIES}

Owing to technical limitations, no consensus has been reached regarding rehabilitative methods in experimental animals. Interventional studies targeting hindlimb and gait function are predominantly conducted in rodents, while few studies have used minipigs, felines, and non-human primates. Many of these studies applied the thoracic cord injury model and implemented physical training including hindlimb motion due to the advantages of more accessible assessment and a higher survival rate, together with the applicability of various $\mathrm{SCl}$ types and their severity. Quadrupedal treadmill training was adopted in most mouse studies, while both bipedal and quadrupedal treadmills were used in rat models. Body-weight supporting apparatus is frequently applied to enable the training in animals with severe impairment. Cycling, swimming, and climbing training routines were also sometimes used in rodents. The most suitable rehabilitative method might differ between models. Quadrupedal training is called physiological gait training because it enables coordination between the forelimbs and hindlimbs. A validated and standardized training protocol was recently reported in moderate contusive $\mathrm{SCl}$ model mice ${ }^{20}$. By stimulating reorganization of rostrocaudal spinal inter-neuronal networks, a research group reported better functional recovery with quadrupedal training than with bipedal gait training in a hemisection rat model $^{21}$. On the other hand, bipedal training has advantages because it explicitly trains the paretic limbs even in severe $\mathrm{SCl}$ animal models that tend to use forelimbs more than hindlimbs in quadrupedal gaits ${ }^{18}$, and it may be better suited for investigating the effects on the local spinal network. Cycling training involves rhythmic sensory-motor training and promotes right-left coordination along with range-of-motion exercises ${ }^{22}$. Some researchers used swimming training for incomplete $\mathrm{SCl}$ model rats because it is a natural behavior of this species. Although it requires simple apparatus, controlling an appropriate exercise load is difficult. Importantly, specific training paradigms to encourage voluntary stepping are more effective than entirely passive stepping ${ }^{18,23}$. Instrumental training promotes activity-based learning and suppresses maladaptive plasticity ${ }^{24}$. By contrast, while it remains controversial, some reports suggest there are unfavorable training conditions that may lead to the development of hypersensitivity, such as delayed initiation of running wheel training resulting in aberrant nociceptive plasticity for cervical hemi-contusion model rats $^{25}$ and early initiation of passive mechanical training for moderately thoracic cord contusion model rats $^{26}$. In addition, researchers have reported a trade-off relationship, the deterioration of a specific function secondary to other training targeting functions, between locomotor and forelimb dexterity tasks in rats with cervical dorsolateral quadrant lesion 27,28 and between standing and stepping abilities in rats with dorsal rhizotomy ${ }^{29}$. This emphasizes the importance of designing optimal therapeutic strategies corresponding to the impairment model ${ }^{22}$.

Forelimb functional recovery in animals with cervical $\mathrm{SCl}$ has also been investigated. Although gait analysis, climbing tasks, the cylinder test, grip strength, and object manipulation, including food-pellet reaching tasks, are applied as assessment measures, reaching tasks require training to evaluate the acquisition of dexterity $^{30-35}$. A single-pellet reaching task, Whishaw reaching, involves horizontal reaching training, and a clear relationship between training intensity and improvement was recently shown in rats with cervical dorsolateral quadrant lesion ${ }^{36}$. The modified Montoya staircase task involves training for perpendicular reaching of each hand independently ${ }^{37}$. Compared with gait training, such task-specific training has advantages including a lack of confounding by off-task home cage self-training that overrides the training effect ${ }^{38}$. In addition, forelimb function has been actively investigated not only with $\mathrm{SCl}$ models, but also in the context of the specific contribution of a tract(s) and propriospinal neurons ${ }^{39}$.

Although the number of rehabilitative training animal models is still limited ${ }^{40}$, a growing number of studies have demonstrated functional recovery induced by rehabilitative training. With regard to motor function, a variety of changes have been reported, including regeneration and reorganization of intra-spinal ${ }^{27,41-43}$ and spinal descending circuits ${ }^{44}$, enforcement of synaptic function $^{45}$, axonal regeneration, and exercise-dependent plasticity ${ }^{46}$, improvements of motor control through the restoration of spinal inhibitory capacity ${ }^{18,47}$, sensory-motor integration, and supraspinal control ${ }^{48}$. Expression of various neurotrophic factors ${ }^{49,50}$, growth factors ${ }^{12,51}$, and both excitatory and inhibitory molecules $^{18,47}$ has been suggested to be involved in these changes. Neurotrophic factors have been proposed to promote neural plasticity, vascularization, and neuroprotection ${ }^{8}$. Furthermore, treadmill training reportedly promotes proliferation and migration of ependymal cells, which are considered to be a source of neural stem cells, in thoracic cord clip-compression model rats ${ }^{52}$. Although it is reported that ependymal cells do not proliferate after injury in the adult human spinal $\operatorname{cord}^{53}$, another group reported that they are activated in some humans of a certain $\mathrm{age}^{54}$ and in animal models including mouse transection $\mathrm{SCl}^{55,56}$. A similar beneficial effect might be induced by rehabilitation in combination with stem cell transplantation.

Another critical role of rehabilitation is to prevent and ameliorate the negative impact of disuse due to impairments ${ }^{57}$. Muscle volume and function decrease after $\mathrm{SCl}$, and the dominant muscular fiber type changes ${ }^{58}$. Importantly, disuse muscle atrophy is also reported to promote motor neuronal degeneration ${ }^{59}$. Moreover, although it is often overlooked in preclinical studies of chronic $\mathrm{SCl}$, disuse-induced functional deterioration is speculated to suppress or even mask the beneficial effect of specific treatments ${ }^{11}$.

\section{METHODOLOGY OF REGENERATIVE REHABILITATION IN PRECLINICAL STUDIES}

Although rehabilitation is commonly performed after cell therapy in human patients, preclinical studies are particularly important for the following purposes: (1) to assess the synergistic effect of transplantation and rehabilitation and (2) to broaden the applications in patients with chronic $\mathrm{SCl}$ in whom stem cell therapy is ineffective when applied alone ${ }^{2}$. Very few preclinical studies have investigated the combinatorial effects of stem cell therapy and rehabilitation regardless of chronicity (Table 1, Supplementary Table 1). Most studies targeted acute and subacute $\mathrm{SCl}$, and only one or two research groups have investigated combinatorial treatment strategies, except for observational studies incorporating very few animals ${ }^{10,11,22}$. Rehabilitation training was usually initiated 2-7 days after transplantation, while the training period varied from 4 weeks to several months. A frequency of 5-7 days per week is adopted in most regimens, although a 3-day regimen was tested for rats with mild thoracic cord contusion $\mathrm{SCl}$ in an earlier study ${ }^{60}$. The time duration varied from 20 to $60 \mathrm{~min}$ per day in most studies, but training for a shorter duration was applied in a few cases ${ }^{61}$. Some researchers incorporated a pretraining period of $\sim 1$ week before the transplantation procedure and the main training period followed this. The purpose of pretraining is to recondition the 
Table 1. $\mathrm{SCl}$ rehabilitation in preclinical studies on stem cell therapies.

Study

2021 Sun W.M. ${ }^{70}$, Rhesus monkeys, $N=4$. Acute Tp (0 DPI), T8 hemisection

2021 Prager J. ${ }^{71}, \mathrm{WH}$ rats, $N=23$. Acute Tp (0 DPI), C3 dorsal column crush

2020 Younsi A. ${ }^{67}, \mathrm{WH}$ rats, $N=70$. Subacute Tp (10 DPI), C6 moderate contusion

2020 Dugan E.A. ${ }^{74}$, SD rats, $N=48$. Late subacute Tp (28 DPI), T6-7 clip contusion

2020 Massoto T.B. ${ }^{66}$, C57 B6j mice, $N=40$. Subacute Tp (7 DPI), T9 clip contusion

2018 Thornton MA. ${ }^{104}$, SD rats, $N=10$. Acute Tp (0 DPI), T6-7 transection

2018 Tashiro S. $^{10}$, C57 B6j mice, $N=45$. Chronic Tp (49 DPI), T9 severe contusion

2017 Theisen C.C. ${ }^{22}$, SD rats, $N=45$. Chronic Tp (42 DPI), Late subacute rehab (35 DPI) T12 transection

2016 Nicola F.C. ${ }^{61}$, WH rats, $N=54$. Acute Tp (0 DPI), T9 moderate contusion

2016 Tashiro S. ${ }^{11}$, C57 B6j mice, $N=80$. Chronic Tp (49 DPI), T9 severe contusion

2016 Sachdeva R. ${ }^{63}$, SD rats, $N=45$. Acute Tp (0 DPI), T12 transection

2015 Dugan E.A. ${ }^{105}$, SD rats, $N=64$. Acute Tp (0 DPI), T10 transection

2014 Hwang D.H. ${ }^{12}$, SD rats, $N=184$. Subacute Tp (7 DPI), T9 moderate contusion

2013 Sun T.65, SD rats, $N=40$. Subacute Tp (14 DPI), T10 moderate contusion

2011 Takeoka A. ${ }^{64}$, WH rats, $N=41$. Acute Tp (0 DPI), T9 transection

2008 Kubasak M.D. ${ }^{62}$, WH rats, $N=38$. Acute Tp (0 DPI), T9 transection

2008 Carvalho K.A.T. ${ }^{106}$, WH rats, $N=48$. Acute Tp (2 DPI), T9/ Swimming with support; $60 \mathrm{~min} /$ day, $6 \mathrm{~d} / \mathrm{wk}$, for 6 wks.

10 mild contusion

2006 Yoshihara $\mathrm{H}^{60}$, SD rats, $N=26$. Subacute Tp (9 DPI), T9-10 mild contusion

2006 Lynskey J.V. ${ }^{107}$, SD rats, $N=84$. Subacute Tp (14 DPI), C5/6 over-hemisection

2005 Keyvan-Fouladi N. ${ }^{72}$, Albino Swiss rats, $N=46$. Chronic Tp (56 DPI), C1/2 corticospinal tract lesion

2005 Ruitenberg M.J. ${ }^{108}$, Fischer rats, $N=29$. Chronic Tp (56

DPI), C4 dorsal hemisection

2003 Keyvan-Fouladi N. ${ }^{109}$, Albino Swiss rats, $N=54$. Chronic Tp (56 DPI), cervical-dorsal hemisection.
Rehabilitation

Regular stretching, standing with supportive chair, positioning, chair supported investigated.

Forepaw reaching; started at 7 DPI, 1 h/day, 5 d/wk, for 8 wks. Untrained condition was not investigated.

Quadrupedal treadmill; started at DPI, $20 \mathrm{~min} /$ day, for 6 wks.

Quadrupedal 8 degree incline treadmill in the ramping protocol; started at 5 or 35 $\mathrm{DPI}, 20 \mathrm{~min} /$ day, $5 \mathrm{~d} / \mathrm{wk}$, for $4 \mathrm{wks}$.

uadrupedal treadmill; started at $14 \mathrm{DPI}, 10 \mathrm{~min} /$ day, $3 \mathrm{~d} / \mathrm{wk}$, for $8 \mathrm{wks}$.

Climb training with $40 \mathrm{~Hz}$ ES at a $95 \%$ of motor threshold; started at $28 \mathrm{DPI}, 20 \mathrm{~min} /$ day, 3/week, for 6 mo. Conditions without training or ES were not evaluated.

Bipedal treadmill; started at $52 \mathrm{DPI}, 20 \mathrm{~min} /$ day, $5 \mathrm{~d} / \mathrm{wk}$, for 8 wks. Pretraining for 1 wk started at $42 \mathrm{DPI}$

Cycling exercise: $30 \mathrm{~min} /$ day; the Delayed group started at 35 DPI for 6 wks, Acute group started at $5 \mathrm{DPI}$ for 10 wks.

Quadrupedal treadmill; started at $3 \mathrm{DPI}, 10 \mathrm{~min} /$ day, $5 \mathrm{~d} / \mathrm{wk}$, for 6 wks. Pretraining for 1 wk

Bipedal treadmill; started at $52 \mathrm{DPI}, 20 \mathrm{~min} /$ day, $5 \mathrm{~d} / \mathrm{wk}$, for 8 wks. Pretraining for 1 wk from 42 DPI

Cycling exercise at $45 \mathrm{rpm}$; started at $5 \mathrm{DPI}, 30 \mathrm{~min} /$ day, $5 \mathrm{~d} / \mathrm{wk}$, for 4 wks.

Passive quadrupedal cycling; started at 5 DPI, $60 \mathrm{~min} /$ day (two 30-min sessions with a 10 -min rest), 5 days/wk, for 12 wks.

Quadrupedal treadmill; started at $10 \mathrm{DPI}, 60 \mathrm{~min} /$ day, $6 \mathrm{~d} / \mathrm{wk}$, for 8 wks. Pretraining for $1 \mathrm{wk}$

Bipedal treadmill; started at $21 \mathrm{DPI}, 20 \pm 10 \mathrm{~min} /$ day, for $10 \mathrm{wks}$.

Bipedal step and treadmill gait; started at $14 \mathrm{DPI}, 20 \mathrm{~min} /$ day, for $7.5 \mathrm{mo}$.

Bipedal treadmill stepping; 5 min in the first week, and increased by 5 min each week up to $20 \mathrm{~min} /$ day, for $6 \mathrm{mo}$ in total. Untrained condition was not investigated histologically.

Passive hindlimb bicycling at $45 \mathrm{rpm}$; started at 11-12 DPI, $60 \mathrm{~min} /$ day (two 30-min sessions with a 10-min rest), $3 \mathrm{~d} / \mathrm{wk}$, for $3 \mathrm{mo}$.

Direct skilled target reaching; started at $42 \mathrm{DPI}, 5-10 \mathrm{~min}$, for 5 days. Untrained condition was not investigated.

Direct forepaw reaching; started at $59 \mathrm{DPI}, 50$ retrieval/limb, 3/wk, for 8 wks. Untrained condition was not investigated.

Direct forepaw reaching; started at $70 \mathrm{DPI}, 3 / \mathrm{wk}$, for $10 \mathrm{wks}$. Untrained condition was not investigated.

Direct forepaw reaching; started at $59 \mathrm{DPI}, 50$ retrieval/limb, 3/wk, for $8 \mathrm{wks}$. Untrained condition was not investigated.

DPI days post injury, ES epidural stimulation, SD rats Sprague Dawley rats, $T p$ transplantation, WH rats Wistar Hannover rat.

animals' body function after a long period of low activity following injury and to habituate the animals for training ${ }^{10-12,62}$. However, to our knowledge, none of these methods is standardized in terms of appropriate load, duration, and methodology.

In the early study of the combinatorial treatment in 2006, Yoshihara et al. ${ }^{60}$ did not observe any remarkable effect on rats with mild contusive thoracic $\mathrm{SCl}$ who received a subacute intramedullary injection of bone marrow-derived MSCs when hindlimb cycling exercise was applied at a frequency of three times per week. In 2016, Sachdeva et al. ${ }^{63}$ applied a similar training method with a higher frequency (five times per week) in spinal cord-transected rats who received a peripheral nerve graft.
They found that cycling exercise strongly facilitated regeneration by propriospinal, but not sensory, neurons, accompanied by increases in mRNA expression of regeneration-associated genes. Regarding other studies that applied minimal doses, Nicola et al. ${ }^{61}$ did not find any additional effect of $10 \mathrm{~min} /$ day training on rats with mild thoracic SCl. Kubasak et al. ${ }^{62}$ applied incremental doses from 5 to $20 \mathrm{~min} /$ day and showed that functional recovery was significantly enhanced in a transection model. These results imply that rehabilitative treatments used in combination with stem cell therapy have a minimum dose threshold and that an appropriate method or strategy is required to elicit beneficial effects. Further 
investigations are needed to determine the appropriate dose, intensity, and training method for each $\mathrm{SCl}$ model.

\section{MECHANISMS OF REGENERATIVE REHABILITATION IN PRECLINICAL STUDIES}

With regard to the behavioral, histological, and functional aspects of hosts with acute and subacute $\mathrm{SCl}$, Takeoka et al. reported that partial body-weight-supported treadmill training (BWSTT) gait exercise enhanced the effect of OEC grafts in super acute thoracic cord-transected rats. They observed that combinatorial treatments induced a fourfold increase in regenerating axons within the caudal stump of the transected spinal cord, and consequently improved hindlimb function and electrophysiological states. In addition, they found that the combinatorial effect was preserved after re-transection of the spinal cord, indicating that regenerative rehabilitation promoted the reorganization of lumbosacral circuits $^{64}$. Sun et al. subacutely transplanted both OECs and Schwann cells into a rat with moderate contusion injury and performed bipedal BWSTT. While the activity of astrocyte-like OECs at the lesion site was not modified, they found that treadmill training significantly contributed to increased serotoninergic activity within lumbar enlargement. Locomotor function was significantly greater in the trained groups, and the effect was greater in the group that received combinatorial therapy than in the trainingonly group ${ }^{65}$. Hwang et al. acutely transplanted NS/PCs into the moderately contused rat spinal cord and then performed bipedal BWSTT for 8 weeks. The combinatorial approach significantly promoted graft survival and differentiation more into neurons and oligodendrocytes, which correlated with greater functional recovery than in the control groups. It also enhanced tissue protection, myelination, and restoration of serotonergic fiber innervation in the lumbar spinal cord, which the authors attributed to reduced stress caused by active oxygen or active nitrogen through insulin-like growth factor (IGF)-1 signaling ${ }^{12}$. In addition, they showed that combined rehabilitation contributed to upregulation of brain-derived neurotrophic factor (BDNF), glial cell line-derived neurotrophic factor (GDNF), and neurotrophic factor 3 (NT-3). Massoto et al. performed MSC transplantation in the acute phase and treadmill training for 8 weeks in mice with moderate clip-compression injury. They reported better functional recovery together with a larger area of white matter, more myelinated fibers, and fewer microcavitations and degenerating nerve fibers, along with significantly increased NT-4 expression ${ }^{66}$. Younsi et al. subacutely transplanted NS/PCs into cervical contusive lesions of rats and then performed quadrupedal treadmill training for 6 weeks. Combined rehabilitation enhanced graft survival and promoted differentiation into neurons and oligodendrocytes. They further demonstrated better functional recovery together with improved myelination, descending tract regeneration, and tissue sparing following combinatorial treatment ${ }^{67}$. Sachdeva et al. performed cycling training in combination with super acute peripheral nerve grafting in a rat transection model. They found that exercise enhanced propriospinal neuronal regeneration and mRNA expression of growth-associated protein 43 (GAP43), $\beta$-actin, and Neuritin, which are responsible for neuronal regeneration, while no remarkable effect was observed on sensory neurons ${ }^{63}$. The mechanisms are briefly illustrated in Fig. 1.

Only some of the studies that performed the combinatorial treatment with transplantation and rehabilitation focused on training-specific effects. Consequently, these studies sometimes did not compare trained and untrained or less-trained animals. This was particularly true of studies of forelimb function that utilized pellet reaching tasks; training was usually implemented just as part of the assessment only before $\mathrm{SCl}^{68}$ or for a short duration before every assessment ${ }^{69}$. Whereas other studies implemented the task as regular rehabilitative training after transplantation, there was no untrained groups ${ }^{70-72}$. Thus, to our knowledge, the specific contribution of regenerative rehabilitation to forelimb functional recovery has not been reported, and the findings described above were all obtained with gait training. Overall, the mechanism of regenerative rehabilitation has not been completely elucidated.

\section{REGENERATIVE REHABILITATION FOR THE REFRACTORY STATE OF DELAYED (LATE SUBACUTE TO CHRONIC) PHASE SCI}

A consensus study concluded that 6 weeks is the minimum timeframe required to reach the chronic phase of $\mathrm{SCl}$ in the rodent model $^{73}$. While combinatorial treatment with rehabilitation is a

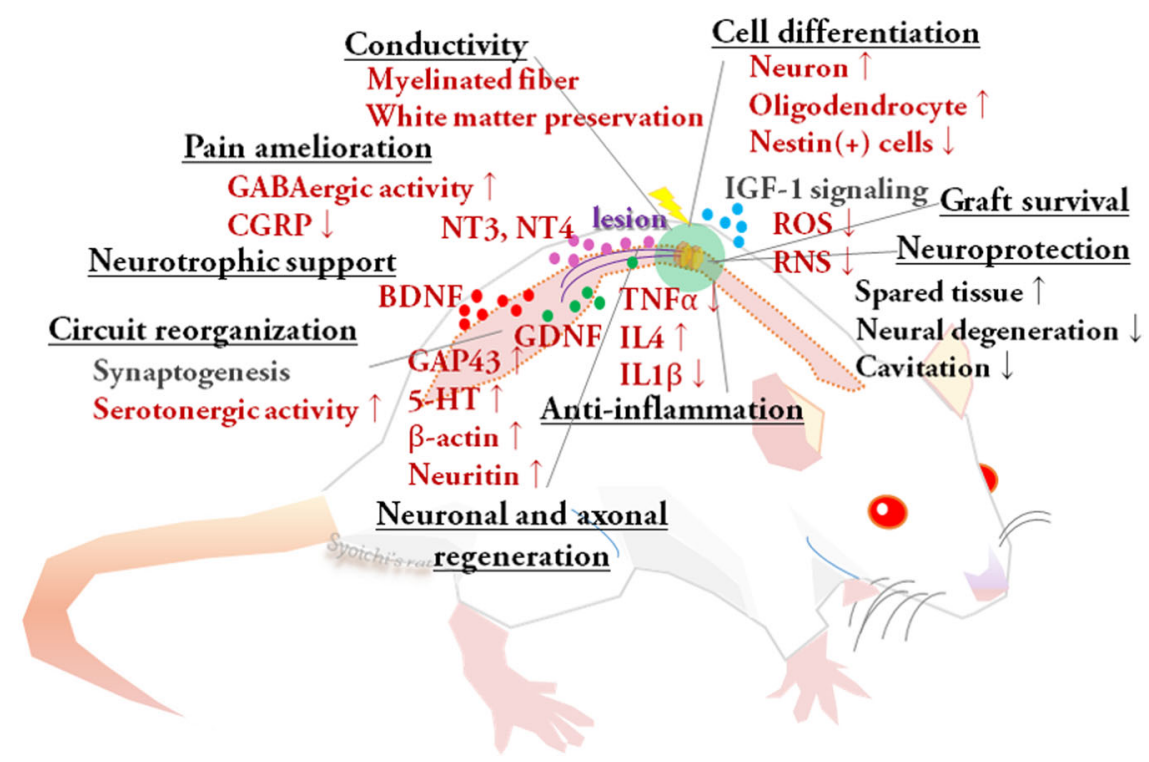

Fig. 1 Mechanisms of regenerative rehabilitation revealed in preclinical studies. The mechanisms of regenerative rehabilitation are briefly summarized in this scheme. Studies lacking comparisons of trained and untrained groups and studies including only a few animals were excluded. BDNF brain-derived neurotrophic factor, CGRP calcitonin gene-related peptide, GAP43 growth-associated protein 43, GDNF glial cell line-derived neurotrophic factor, IGF-1 insulin-like growth factor, IL $1 \beta / 4$ interleukin $1 \beta / 4$, GABA gamma aminobutyric acid, NT-3/-4 neurotrophic factor $3 / 4$, RNS reactive nitrogen species, ROS reactive oxygen species, TNFa tumor necrosis factor $\alpha, 5$-HT 5-hydroxytryptamine. 
favorable treatment option, only two studies investigated combinatorial treatment with cell transplantation and rehabilitation in the chronic phase of $\mathrm{SCl}$ if the 6-week rule is strictly applied $^{10,11}$. Tashiro et al. ${ }^{10,11}$ studied motor and sensory recovery following transplantation in combination with rehabilitation in the chronic phase. They performed NS/PC transplantation followed by bipedal BWSTT for 8 weeks in severe contusive thoracic SCI mouse models. The mice displayed significant locomotor recovery, which corroborated the synergistic effect of the combinatorial treatment on axonal regeneration and synaptogenesis, as well as an increase in neuronal differentiation of the transplanted cells. The additive effects on immunohistological changes imply that serotoninergic activity was enhanced by transplantation and GABAergic activity was restored by rehabilitation ${ }^{11}$. They further reported amelioration of thermal allodynia and coarse touch-pressure hyperalgesia, and a reduction of calcitonin gene-related peptide-positive fibers, which function in the transmission of pain, together with upregulation of GABAergic activity in the posterior horn ${ }^{10}$. Although they demonstrated significant functional recovery upon combinatorial therapy, no pertinent differences were observed between the combinatorial treatment and rehabilitation alone groups. Thus, they concluded that an additional treatment(s) is needed to use stem cell therapies in patients with chronic $\mathrm{SCl}^{11}$. Theisen et al. ${ }^{22}$ investigated the effects of peripheral nerve grafting at 42 days post injury (DPI) in combination with cycling exercise in T12-transected rats and reported that spinal axon regeneration was enhanced after exercise. No significant difference was detected between the two exercise periods, namely, 6 weeks starting at $35 \mathrm{DPI}$ and 10 weeks starting at $7 \mathrm{DPI}$. Although these outcomes were due to a combination of chronic transplantation and acute or late subacute rehabilitative interventions, we would classify the latter as chronic regenerative rehabilitation. The difference between late subacute and chronic phase $\mathrm{SCl}$ has not been determined in terms of rehabilitation studies. A study by the same laboratory further compared the results of chronic peripheral nerve grafting with acute interventions $^{63}$ and concluded that exercise has no long-lasting or cumulative effect in terms of axonal regeneration.

Regarding late subacute interventions, Dugan et al. investigated the role of the local spinal inhibitory circuit upon GABAergic neuronal progenitor cell transplantation in thoracic cord clipcompression rat models. Cells were transplanted into the lumbar enlargement excluding the injury site at $28 \mathrm{DPI}$, and treadmill quadrupedal training with an incline of $8^{\circ}$ was performed for 4 weeks. They demonstrated that neuropathic pain assessed by allodynia, hyperalgesia, and ongoing pain was ameliorated upon reduction of inflammation, as represented by upregulation of IL4 and downregulation of tumor necrosis factor $a$ and interleukin $1 \beta$, together with increased BDNF expression. Moreover, combined rehabilitation in both the subacute and chronic phases significantly promoted the restoration of GABAergic activity, while transplantation alone did not induce remarkable changes ${ }^{74}$. These studies imply that the effects of combinatorial treatment with rehabilitation and stem cell transplantation in the chronic phase are not inferior to those in the subacute phase with regard to sensory recovery, but the effects of transplantation on motor function decrease over time.

\section{PROGRESS OF NEUROREHABILITATION IN CLINICS}

Joint range-of-motion exercise, paretic and non-paretic muscle strengthening, systemic physical capacity training, and practice of basic motions, including gait and transfer, are clinically applied as conventional rehabilitation ${ }^{75}$. Great efforts have been made to develop more effective rehabilitative treatments for patients with $\mathrm{SCl}$. Researchers have reported that intensive overground walking together with training of balance function ${ }^{76}$, BWST $^{77}$, and training of hand and reaching function ${ }^{78}$ effectively improve body function of $\mathrm{SCl}$ patients. It is noteworthy that multimodal rehabilitation can induce functional recovery even in patients with chronic motor-complete $\mathrm{SCl}^{79}$. It is suggested that the central nervous system (CNS) reorganizes itself during the acquisition, retention, and consolidation of motor skills. This concept is summarized as neurorehabilitation and neuroplasticity ${ }^{80}$. There might be such significant overlap between the chief mechanisms underlying the effects of stem cell therapies and neurorehabilitation that these two treatments show synergism ${ }^{8,16}$. Although most neurorehabilitative treatments are non-invasive, some require a neurosurgical procedure to implant epidural electrodes or an intrathecal drug administration system ${ }^{81}$.

Studies have reported the effects of various "peripheral" neurorehabilitative methods that target the peripheral sensorimotor system. Functional electrical stimulation (FES), including neuromuscular electrical stimulation (NMES), is a method to assist the impaired muscular function of patients using electrical stimulation. NMES assists the voluntary movements of patients by enhancing the intent of movements via timely and tuned electrical stimulation. It is utilized for the training of both gait and upper limb activities ${ }^{82}$. BWSTT and robot-assisted gait training (RAGT) improve the gait of patients in a more accessible, faster, and safer manner than regular overground training ${ }^{83}$. Some types of RAGT include additional functions such as $\mathrm{FES}^{84}$. Brain-computer interface is a new technology that connects specific brain signals of thoughts, perceptions, and motor intent to the output device, which provides a specific response that rewards functional training or a compensatory response to the impaired body function ${ }^{85}$.

On the other hand, the "central" neurorehabilitative method targeting the CNS has also been investigated with various means and strategies. Preclinical studies showed that direct current stimulation induces migration and proliferation of neural progenitor cells together with upregulation of neurotrophic factors ${ }^{86}$. In addition, both non-invasive transcutaneous and minimally invasive spinal/brain stimulation has been developed owing to their direct and harmless neuromodulatory properties ${ }^{87,88}$. Moreover, a series of studies revealed that combinatorial treatment with intrathecal medication and monoamine agonists enhances and manipulates the effect of electrical stimulation ${ }^{89}$. The brainspine interface was recently reported in a primate model in which an electroencephalograph was applied to control epidural stimulation patterns ${ }^{90}$. Utilizing these multimodal neurorehabilitative approaches, Asboth et al. reported a novel rehabilitative treatment in which body-weight-supported RAGT was performed in combination with spinal epidural stimulation and treatment with serotoninergic and dopaminergic agonists in a preclinical study. They further observed reorganization of the efferent circuit cortico-reticulospinal circuit, which caused rerouting of cortical projections and contributed to functional recovery ${ }^{44}$.

\section{REGENERATIVE REHABILITATION IN CLINICAL STUDIES: DIFFERENCE BETWEEN ACUTE-TO-SUBACUTE PHASE AND CHRONIC PHASE}

Several clinical studies of stem cell-based regenerative treatments with cellular or tissue grafting for human patients with $\mathrm{SCl}$ have been conducted worldwide. This section summarizes the features of rehabilitation in five studies of acute $\mathrm{SCl}$, four studies of subacute $\mathrm{SCl}, 33$ studies of chronic $\mathrm{SCl}$, and nine studies in which the chronicity was not specified (Tables 2 and 3, Supplementary Tables 2 and 3). A group reported that intensive neurorehabilitation was applied for children who underwent transplantation involving surgical procedure ${ }^{91}$. It is noteworthy that only standard/conventional rehabilitation training was performed in studies of patients with acute and subacute $\mathrm{SCl}$. This might be because preclinical research showed that stem cell therapies only induce significant functional improvement in the early phases. 
Table 2. SCl rehabilitation in clinical studies on stem cell therapies involving acute/subacute patients.

Study information

2020 Chen W. ${ }^{100}$, Phase I, $N=7$. Acute $\mathrm{SCl}$, implantation, autologous bone marrow mononuclear cells loaded into NeuroRegen scaffolds

2020 Sharma A. ${ }^{110}$, Open-label, $N=180$. Subacute to chronic SCI, intrathecal injection, autologous bone marrow mononuclear cells

2017 Anderson K.D. ${ }^{11}$, Phase I, $N=6$. Subacute SCl, intramedullary injection, autologous Schwann cells *A study to develop a home program was derived from this trial ${ }^{101}$

2013 Liu J. ${ }^{112} N=22$., Subacute to chronic SCl, intrathecal injection, umbilical cord mesenchymal stem cells

2007 Yoon S.H. ${ }^{113}$, Phase: I/II, $N=35$. Acute-to-chronic SCl: acute $(<14$ DPI): $N=17$, Subacute ( $\geq 14 \mathrm{DPI},<8$ weeks): $N=6$, Subacute-Chronic

( $\geq 8$ weeks): $N=12$ Intramedullary injection, autologous bone marrow cell

Ravinovich S.S. ${ }^{114}$, Knoller N. ${ }^{115}$, Shin J.C. ${ }^{116}$, Karamouzian S. ${ }^{117}$, Chhabra A standard rehabilitation H.S. ${ }^{118}$, Satti H.S. ${ }^{119}$, Hur J.W. ${ }^{120}$, Bansal H. ${ }^{121}$, Xiao Z. ${ }^{122}$

Pal R. ${ }^{123}$, Attar A. ${ }^{124}$, Kumar A.A. ${ }^{125}$, Saito F. ${ }^{126,127}$, Jones L.A.T. ${ }^{128}$, Lammertse D.P. ${ }^{129}$

$D P I$ days post injury, $S C l$ spinal cord injury, $T p$ transplantation.
Rehabilitation

6 months of standard rehabilitation including routine care, hyperbaric oxygen therapy, neurotrophic therapy, acupuncture, neuromuscular electrical stimulation therapy, upper limb muscle strength training, selfcare training, etc.

Neurorehabilitation, including physiotherapy, occupational therapy, psychological interventions, and aquatic therapy. A home program under the supervision of professionals was recommended.

An inpatient standard medical rehabilitation, for 3-5 weeks pre-Tp, and for $6.6 \pm 2.1$ weeks post-Tp; $3 \mathrm{~h} /$ day, $5 \mathrm{~d} / \mathrm{wk}$.

A systemic individualized physical therapy

An active rehabilitation

No description about rehabilitation
Therefore, researchers have no specific obligation to deliver stem cell therapy in combination with an intensive strategy or a neurorehabilitative approach using an advanced device, i.e., regenerative rehabilitation in a narrow sense.

Since stem cell therapy has fewer effects on chronic SCl, combining rehabilitation attracts wide attention with its feasibility over the success in preclinical studies. Many research groups have implemented specific combined rehabilitative approaches. Furthermore, Huang et al. ${ }^{92}$ reported that the quality and quantity of rehabilitation influenced the long-term outcome in patients who underwent OEC transplantation, although they did not investigate the threshold. To our knowledge, this is the only study investigating the relationship between functional recovery and the characteristics of rehabilitation after stem cell therapy.

Rehabilitative interventions are often applied both before and after stem cell treatment in clinical studies involving patients with chronic SCl. We named the former "pre-rehabilitation" and the latter "post-rehabilitation". Usually, only conventional rehabilitative methods are applied as pre-rehabilitation. Pre-rehabilitation can be categorized as mobilization of disuse-provoked impairments such as weakened and contracted muscles, homogenization of the status of patients before stem cell treatment, and identification of responsiveness to rehabilitation, which is far feasible than stem cell therapies. It is noteworthy that a study even excluded cases that showed a possibility of recovery with rehabilitation ${ }^{93}$.

\section{STRATEGIES OF REGENERATIVE REHABILITATION APPLIED IN THE CLINICAL STUDIES}

Rehabilitative strategies appeared in regenerative studies can be characterized into six large groups:

1. The majority of groups seemed to apply only conventional rehabilitation. No rehabilitative protocol was provided in most cases $^{94-96}$.

2. Intensive rehabilitation with the conventional method was also frequently applied. A massive dose of rehabilitation of $>4-6 \mathrm{~h}$ per day was applied for a remarkably long duration exceeding 24 weeks up to 1 year ${ }^{97}$.

3. Several researchers chose a traditional, but specific, rehabilitative approach to maximize the effect of stem cell therapy. The principles of these approaches include facilitation of physical exercise ${ }^{98,99}$, early induction of training for essential motion including gait and $\mathrm{ADL}^{14,15}$, sensory training $^{14,15,98}$, and the facilitation method ${ }^{13}$.

4. It was not rare for advanced rehabilitation, including $\mathrm{FES}^{14,93,99}$, NMES ${ }^{100}$, Upper body circuit resistance training $^{101}$, BWST $^{14,15,99}$, and RAGT ${ }^{14}$, to be implemented.

5. Physical treatments including electrical field, ultrasound, and temperature gradients were also applied to induce mechanotransductive effects ${ }^{8,15,99}$.

6. Home-based aerobic and strengthening program using dumbbell, resistance band, and upper limb ergometer ${ }^{101}$.

Although it is difficult to know the exercise load in home-based training, Maher et al. ${ }^{101}$ utilized the talk-test to determine an intensity that made speaking uncomfortable in maintaining the appropriate intensity level. We suggest that the second, third, fourth, fifth, and sixth groups are regenerative rehabilitation in a narrow sense, whereas the first group is combined rehabilitation, i.e., regenerative rehabilitation in a broad, but not a narrow, sense.

According to the mechanism revealed in preclinical and clinical studies, the roles of regenerative rehabilitation can be categorized as (i) conditioning/reconditioning, (ii) functional training, and (iii) physical exercise. Conditioning and reconditioning mainly target physical problems such as contracture, muscle atrophy, and cardiopulmonary deconditioning, which are derived from disuse due to $\mathrm{SCl}$ sequelae. They are even more important in the chronic phase because of the long deconditioning period ${ }^{2,57,102}$. Functional training will be the core element of regenerative rehabilitation. Although researchers have elucidated various micromechanisms via which $\mathrm{SCl}$ rehabilitation, in general, promotes functional recovery at the molecular, synaptic, local circuit, tract, and even cortical levels, the number of evidence showing interaction with residual tissue or transplanted cells is few in the field of regenerative rehabilitation. It is remarkable there has been no study investigating the effect brought about by rehabilitation regarding forelimb-hand function. Physical exercise is crucial to upregulate neurotrophic factors. These factors have remarkable effects including neuroprotection, especially in the acute phase, modification of proliferation of transplanted cells and their 
Table 3. $\mathrm{SCl}$ rehabilitation in clinical studies on stem cell therapies for chronic patients.

Study information

Rehabilitation

2021 Gant K.L. ${ }^{130}, N=8$ Injection (cavity-filling), autologous human SCs

pper extremity circuit resistance training; 3 /wk Conditioning of lower extremities: FES and cycle ergometer; 2 /wk BWSTT with robot for AIS A or $\mathrm{B}$, or overground locomotor skill for AIS C; biweekly.

2016 Zhu H. ${ }^{97}$, Yao L. ${ }^{131}, N=28$ Intramedullary injection, umbilical cord Intensive locomotor training; started at $14 \mathrm{POD}, 6 \mathrm{~h} / \mathrm{day}, 6 \mathrm{~d} / \mathrm{wk}$, for blood-derived mononuclear cells

2016 Oh S.K. ${ }^{93}$, Phase III, $N=16$ Subdural injection, autologous MSCs 3-6 mo

(Pre) included only when no improvements with 3 months rehabilitation (Post) a standardized PT, twice a day, $6 \mathrm{~d} / \mathrm{wk}$, from 7 POD for 4 wks: tilt table ( $30 \mathrm{~min}$ ), mat exercises (assistive ROM, overground functional activities, $30 \mathrm{~min}$ ) FES.

2016 Iwatsuki K. ${ }^{132}, N=8$ Olfactory mucosa grating with scar removal

A standard PT to encourage function below the lesion, enabling walking training as soon as possible; $15 \mathrm{~h} / \mathrm{wk}$ for 8 wks preoperatively, $48 \mathrm{wks}$ postoperatively.

2016, 2013 Oraee-Yazdani S. ${ }^{133}, N=8$. Intrathecal injection, autologous BM-MSCs, and SCs

(Pre) to continue the same rehabilitation program for 6 mo to exclude dependency of any improvement to rehabilitation (Post) a regular rehabilitation program

2014 El-Kheir WA. ${ }^{134}$, Phase I-II, $N=70$. Intrathecal injection, autologous PT programs; $1-2 \mathrm{~h}, 3$ /wk. (Pre) no details were provided, (Post) started at BM-derived cells

2014 Mendonça MV. ${ }^{136}, N=14$. Intramedullary injection, BM-MSCs upright posture on a tilt table, and cardiopulmonary training (same description with Kishk NA. ${ }^{135}$ )

Detail not described; started at 7 POD, for $6 \mathrm{mo}, 4 \mathrm{~h} / \mathrm{d}$ in first $2 \mathrm{mo}$, and $2 \mathrm{~h} / \mathrm{d}$ thereafter, 5 /wk

2013 Larson $C A .{ }^{15}, N=13$. Olfactory mucosa grating with scar removal

Intense outpatient PT; $3 \mathrm{~h}$ sessions, 3-5/wk, for 4.6 mo (at least $3 \mathrm{mo}$ (i) pre-gait (weight-bearing, posture, balance, crawling, and standing) and/or gait training (BWSTT and overground gait), (ii) intense therapeutic exercise (repetitive neuromuscular facilitation, mat mobility, strengthening and endurance, whole-body vibration, biofeedback, virtual gaming, and/or musculoskeletal interventions), and (iii) FES cycling or static/dynamic standing frame activities

2013 Dai G. ${ }^{137}$, Phase I-II, $N=40$. Intramedullary injection, autologous BM-MSCs

(Pre) to receive formal rehabilitation during the observation period to exclude the effect of rehabilitation (Post) detail not described

2013 Derakhshanrad N ${ }^{138}$, Phase I, $N=12$. Sural nerve in autologous fibrin coagulum grafting in the syrinx

2013 Tabakow $\mathrm{P}^{98}, N=6$. Intramedullary injection, autologous OECs

(Pre) a standard rehabilitation at least 6 mo (Post) a wheelchair transfer at $48 \mathrm{~h}$ after $\mathrm{Tp}$, and post-rehabilitation resumed

ROM (60 min), locomotor training including treadmill (180 min), sensory training ( $60 \mathrm{~min})$; $4-5 \mathrm{~h} /$ day, $3-5 \mathrm{~d} / \mathrm{wk}$ for 3 mo preoperatively, 24 mo postoperatively.

2010 Kishk NA. ${ }^{135}, N=64$. Monthly intrathecal injection, 6 mo, BM-MSCs

2008, 2011 Seberi $\mathrm{H}^{99}{ }^{139}, N=33$. Intramedullary injection, SCs

General rehabilitation: mat and transfer activities, self-ROM, strengthening ambulation, tilt table, and cardiopulmonary training; $3 / \mathrm{wk}$, for $6 \mathrm{mo}$.

Physical exercise, FES, ultrasonic diathermy and infrared; $3 \mathrm{~h} /$ day, $3 \mathrm{~d} / \mathrm{wk}$ for 6 mo preoperatively, 12 mo postoperatively

2006, 2010 Lima C., ${ }^{14,140}$, Phase I-II, $N=20$. Olfactory mucosa grating with scar removal

Passive assisted ROM and strengthening; $2 \mathrm{~h}$, functional training for balance, posture, standing, and transfers; $2-3 \mathrm{~h}$, pre-gait, and gait activities. BWSTT, Lokomat ${ }^{\circledR}$, BIONT which is an assisted overground walking training, with loading on hips, knees, and feet to promote sensorimotor biofeedback; $2-3 \mathrm{~h} .31 .8 \mathrm{~h} / \mathrm{wk}$, for 34.7 wks preoperatively, and $32.7 \mathrm{~h} / \mathrm{wk}$ for 92 wks postoperatively

Jarocha D. ${ }^{91} /$ Moviglia G.A. ${ }^{13} /$ Cheng H. ${ }^{141} /$ Goni V.G. ${ }^{142} /$ Chernykh E.R., ${ }^{143}$ Huang $\mathrm{H} .{ }^{92} /$ Cheng $\mathrm{L} .{ }^{145}$

Zhu H. ${ }^{97 *}$, Yao L. ${ }^{131 *}$, Wang S. ${ }^{144}$

Thompson F.J. ${ }^{146}$, Wirth ED3rd. ${ }^{147}$, Feron F. ${ }^{148}$, Mackay-Sim A. ${ }^{149}$, Cristante A.F. ${ }^{150}$, Ra J.C. ${ }^{151}$, Wu J. ${ }^{152}$, Frolov A.A. ${ }^{153}$, Rao Y. ${ }^{154}$, Al-Zoubi An intensive neurorehabilitation for 4 wks/Vojta and Bobath neurorehabilitation program/A functional recovery and urinary retention training/A standardized physical rehabilitation program/A regular rehabilitation/A rehabilitation for 6 mo.

No rehabilitation, *: no rehabilitation at one out of two sites No description about rehabilitation A., ${ }^{155}$ Vaquero J. ${ }^{96,156,157}$, Curtis E. ${ }^{94}$, Levi A.D. ${ }^{95,158}$

AIS ASIA impairment scale, BM bone marrow, BWSTT body weight-supported treadmill training, FES functional electrical stimulation, MSC mesenchymal stem cell, OEC olfactory ensheathing cell, POD post-operative day, PT physiotherapy, ROM range of motion, Tp transplantation, SC Schwann cell.

differentiation into neurons and oligodendrocytes, and induction of host neural plasticity ${ }^{12,49,67}$. Thus, physical exercise acts like medication. However, it remains to be elucidated to what degree the functional training and the physical exercise are distinguishable; whereas these two approaches possess a different character, there is still some overlapping. Further investigations are needed.

\section{PERSPECTIVE FOR REGENERATIVE REHABILITATION FOR SCI}

In the field of stem cell regenerative medicine, the role of rehabilitation is becoming increasingly important, with preclinical studies delineating the molecular mechanisms and effects. Studies of combinatorial treatment have reported synergistic effects $^{11,12,64}$. Although stem cell therapy seems to induce only 
limited recovery, the additive effect of regenerative rehabilitation may be key to realizing significant motor and functional recovery following chronic SCl. However, it seems difficult for combinatorial treatments incorporating stem cell therapy and rehabilitation to outperform each individual treatment, particularly in the chronic phase ${ }^{10,11,15}$. Therefore, it is crucial to optimize regenerative rehabilitation based on the mechanisms to maximize the effects of stem cell therapy. In addition, further investigations are needed to discover a way of enhancing recovery.

To date, researchers are pointing out the therapeutic potential to combine stem cell therapy and rehabilitation with pharmacological treatments as represented by neurotrophic factors and reagents against axonal growth inhibitor ${ }^{1,2}$. Combination treatment with emerging novel technologies from the fields of bioengineering or molecular biology has evolvability either. For example, neuromodulation with multimodal therapies including spinal epidural stimulation, brain-computer interface, neuroprosthetics, and pharmacological intervention is expected to restore functional movement upon inducing plasticity of spared circuits and residual projection ${ }^{81}$. On the other hand, in vivo reprogramming technology, which enables the generation of new neurons from non-neuronal cells via reprogramming, was recently developed, which is proposed as a novel regenerative strategy ${ }^{103}$. While no study has combined these groundbreaking technologies with stem cell therapy or rehabilitation, to the best of our knowledge, we believe that such extended regenerative rehabilitative strategies will further broaden the therapeutic potential of $\mathrm{SCl}$.

\section{DATA AVAILABILITY}

Data sharing is not applicable to this article as no datasets were generated or analyzed during the current study.

Received: 19 February 2021; Accepted: 21 October 2021; Published online: 25 November 2021

\section{REFERENCES}

1. Tsuji, O. et al. Concise review: laying the groundwork for a first-in-human study of an induced pluripotent stem cell-based intervention for spinal cord injury. Stem Cells 37, 6-13 (2019).

2. Tashiro, S., Nakamura, M. \& Okano, H. The prospects of regenerative medicine combined with rehabilitative approaches for chronic spinal cord injury animal models. Neural Regen. Res. 12, 43-46 (2017).

3. Okano, H. Strategic approaches to regeneration of a damaged central nervous system. Cornea 30, S15-S18 (2011).

4. Nakamura, M. \& Okano, H. Cell transplantation therapies for spinal cord injury focusing on induced pluripotent stem cells. Cell Res. 23, 70-80 (2013).

5. Nishimura, S. et al. Time-dependent changes in the microenvironment of injured spinal cord affects the therapeutic potential of neural stem cell transplantation for spinal cord injury. Mol. Brain 6, 3 (2013).

6. Kumamaru, H. et al. Therapeutic activities of engrafted neural stem/precursor cells are not dormant in the chronically injured spinal cord. Stem Cells 31, 1535-1547 (2013).

7. Shinozaki, M. et al. Combined treatment with chondroitinase $A B C$ and treadmill rehabilitation for chronic severe spinal cord injury in adult rats. Neurosci. Res. 113, 37-47 (2016).

8. Rando, T. A. \& Ambrosio, F. Regenerative rehabilitation: applied biophysics meets stem cell therapeutics. Cell Stem Cell 22, 306-309 (2018).

9. Ross, H. H. et al. Neural stem cell therapy and rehabilitation in the central nervous system: emerging partnerships. Phys. Ther. 96, 734-742 (2016).

10. Tashiro, S. et al. The amelioration of pain-related behavior in mice with chronic spinal cord injury treated with neural stem/progenitor cell transplantation combined with treadmill training. J. Neurotrauma 35, 2561-2571 (2018).

11. Tashiro, S. et al. Functional recovery from neural stem/progenitor cell transplantation combined with treadmill training in mice with chronic spinal cord injury. Sci. Rep. 6, 30898 (2016).

12. Hwang, D. H. et al. Survival of neural stem cell grafts in the lesioned spinal cord is enhanced by a combination of treadmill locomotor training via insulin-like growth factor-1 signaling. J. Neurosci. 34, 12788-12800 (2014).
13. Moviglia, G. A. et al. Combined protocol of cell therapy for chronic spinal cord injury. Report on the electrical and functional recovery of two patients. Cytotherapy 8, 202-209 (2006).

14. Lima, C. et al. Olfactory mucosal autografts and rehabilitation for chronic traumatic spinal cord injury. Neurorehabil. Neural. Repair 24, 10-22 (2010).

15. Larson, C. A. \& Dension, P. M. Effectiveness of intense, activity-based physical therapy for individuals with spinal cord injury in promoting motor and sensory recovery: is olfactory mucosa autograft a factor? J. Spinal Cord. Med. 36, 44-57 (2013).

16. Yang, B. et al. Strategies and prospects of effective neural circuits reconstruction after spinal cord injury. Cell Death Dis. 11, 439 (2020).

17. Kamelska-Sadowska, A. M., Wojtkiewicz, J. \& Kowalski, I. M. Review of the current knowledge on the role of stem cell transplantation in neurorehabilitation. Biomed. Res. Int. 2019, 3290894 (2019).

18. Tashiro, S. et al. BDNF induced by treadmill training contributes to the suppression of spasticity and allodynia after spinal cord injury via upregulation of KCC2. Neurorehabil. Neural Repair 29, 677-689 (2014).

19. Mason, C. \& Dunnill, P. A brief definition of regenerative medicine. Regen. Med. 3, 1-5 (2008).

20. Shibata, T. et al. Treadmill training based on the overload principle promotes locomotor recovery in a mouse model of chronic spinal cord injury. Exp. Neurol. 345, https://doi.org/10.1016/j.expneurol.2021.113834 (2021).

21. Shah, P. K. et al. Use of quadrupedal step training to re-engage spinal interneuronal networks and improve locomotor function after spinal cord injury. Brain 136, 3362-3377 (2013).

22. Theisen, C. C. et al. Exercise and peripheral nerve grafts as a strategy to promote regeneration after acute or chronic spinal cord injury. J. Neurotrauma 34, 1909-1914 (2017).

23. Lee, C., Won, D., Cantoria, M. J., Hamlin, M. \& de Leon, R. D. Robotic assistance that encourages the generation of stepping rather than fully assisting movements is best for learning to step in spinally contused rats. J. Neurophysiol. 105, 2764-2771 (2011).

24. Hansen, C. N. et al. Sparing of descending axons rescues interneuron plasticity in the lumbar cord to allow adaptive learning after thoracic spinal cord injury. Front. Neural Circuits 10, 11 (2016).

25. Detloff, M. R. et al. Delayed exercise is ineffective at reversing aberrant nociceptive afferent plasticity or neuropathic pain after spinal cord injury in rats. Neurorehabil. Neural Repair 30, 685-700 (2016).

26. Endo, T. et al. Early exercise in spinal cord injured rats induces allodynia through TrkB signaling. Biochem. Biophys. Res. Commun. 381, 339-344 (2009).

27. Girgis, J. et al. Reaching training in rats with spinal cord injury promotes plasticity and task specific recovery. Brain 130, 2993-3003 (2007).

28. Weishaupt, N., Vavrek, R. \& Fouad, K. Training following unilateral cervical spinal cord injury in rats affects the contralesional forelimb. Neurosci. Lett. 539, 77-81 (2013).

29. Edgerton, V. R. et al. Use-dependent plasticity in spinal stepping and standing. Adv. Neurol. 72, 233-247 (1997).

30. Rosenzweig, E. S. et al. Restorative effects of human neural stem cell grafts on the primate spinal cord. Nat. Med. 24, 484-490 (2018).

31. Kobayashi, Y. et al. Pre-evaluated safe human iPSC-derived neural stem cells promote functional recovery after spinal cord injury in common marmoset without tumorigenicity. PLoS ONE 7, https://doi.org/10.1371/journal.pone.0052787 (2012).

32. Suzuki, H. et al. Neural stem cell mediated recovery is enhanced by chondroitinase $A B C$ pretreatment in chronic cervical spinal cord injury. PLOS ONE 12, https://doi.org/10.1371/journal.pone.0182339 (2017).

33. Khazaei, M. et al. GDNF rescues the fate of neural progenitor grafts by attenuating Notch signals in the injured spinal cord in rodents. Sci. Transl. Med. 12, https://doi.org/10.1126/scitranslmed.aau3538 (2020).

34. Jones, I., Novikova, L. N., Wiberg, M., Carlsson, L. \& Novikov, L. N. Human embryonic stem cell-derived neural crest cells promote sprouting and motor recovery following spinal cord injury in adult rats. Cell Transplant. 30, https://doi. org/10.1177/0963689720988245 (2021).

35. Starkey, M. L., Bleul, C., Maier, I. C. \& Schwab, M. E. Rehabilitative training following unilateral pyramidotomy in adult rats improves forelimb function in a non-task-specific way. Exp. Neurol. 232, 81-89 (2011).

36. Fenrich, K. K. et al. Self-directed rehabilitation training intensity thresholds for efficient recovery of skilled forelimb function in rats with cervical spinal cord injury. Exp. Neurol. 339, https://doi.org/10.1016/j.expneurol.2020.113543 (2021).

37. Gallegos, C., Carey, M., Zheng, Y., He, X. \& Cao, Q. L. Reaching and grasping training improves functional recovery after chronic cervical spinal cord injury. Front. Cell Neurosci. 14, 110 (2020).

38. Torres-Espin, A. et al. Eliciting inflammation enables successful rehabilitative training in chronic spinal cord injury. Brain 141, 1946-1962 (2018).

39. Tohyama, T. et al. Contribution of propriospinal neurons to recovery of hand dexterity after corticospinal tract lesions in monkeys. Proc. Natl Acad. Sci. USA 114, 604-609 (2017). 
40. Torres-Espin, A., Beaudry, E., Fenrich, K. \& Fouad, K. Rehabilitative training in animal models of spinal cord injury. J. Neurotrauma 35, 1970-1985 (2018).

41. Ichiyama, R. M. et al. Step training reinforces specific spinal locomotor circuitry in adult spinal rats. J. Neurosci. 28, 7370-7375 (2008).

42. Tahayori, B. \& Koceja, D. M. Activity-dependent plasticity of spinal circuits in the developing and mature spinal cord. Neural Plast. 2012, https://doi.org/10.1155/ 2012/964843 (2012).

43. Takeoka, A., Vollenweider, I., Courtine, G. \& Arber, S. Muscle spindle feedback directs locomotor recovery and circuit reorganization after spinal cord injury. Cell 159, 1626-1639 (2014).

44. Asboth, L. et al. Cortico-reticulo-spinal circuit reorganization enables functional recovery after severe spinal cord contusion. Nat. Neurosci. 21, 576-588 (2018).

45. Petruska, J. C. et al. Changes in motoneuron properties and synaptic inputs related to step training after spinal cord transection in rats. J. Neurosci. 27, 4460-4471 (2007).

46. Houle, J. D. \& Cote, M. P. Axon regeneration and exercise-dependent plasticity after spinal cord injury. Ann. N. Y. Acad. Sci. 1279, 154-163 (2013).

47. Tillakaratne, N. J. et al. Use-dependent modulation of inhibitory capacity in the feline lumbar spinal cord. J. Neurosci. 22, 3130-3143 (2002).

48. Winchester, P. et al. Changes in supraspinal activation patterns following robotic locomotor therapy in motor-incomplete spinal cord injury. Neurorehabil. Neural Repair 19, 313-324 (2005).

49. Gomez-Pinilla, F., Ying, Z., Roy, R. R., Molteni, R. \& Edgerton, V. R. Voluntary exercise induces a BDNF-mediated mechanism that promotes neuroplasticity. J. Neurophysiol. 88, 2187-2195 (2002).

50. Gomez-Pinilla, F. et al. BDNF and learning: evidence that instrumental training promotes learning within the spinal cord by up-regulating BDNF expression. Neuroscience 148, 893-906 (2007).

51. Skup, M. et al. Long-term locomotor training up-regulates trkbfl receptor-like proteins, brain-derived neurotrophic factor, and neurotrophin 4 with different topographies of expression in oligodendroglia and neurons in the spinal cord. Exp. Neurol. 176, 289-307 (2002).

52. Foret, A. et al. Stem cells in the adult rat spinal cord: plasticity after injury and treadmill training exercise. J. Neurochem. 112, 762-772 (2010).

53. Paniagua-Torija, B. et al. Cells in the adult human spinal cord ependymal region do not proliferate after injury. J. Pathol. 246, 415-421 (2018).

54. Cawsey, T., Duflou, J., Weickert, C. S. \& Gorrie, C. A. Nestin-positive ependymal cells are increased in the human spinal cord after traumatic central nervous system injury. J. Neurotrauma 32, 1393-1402 (2015).

55. Barnabe-Heider, F. et al. Origin of new glial cells in intact and injured adult spinal cord. Cell Stem Cell 7, 470-482 (2010).

56. Moreno-Manzano, V. Ependymal cells in the spinal cord as neuronal progenitors. Curr. Opin. Pharm. 50, 82-87 (2020).

57. Bodine, S. C. Disuse-induced muscle wasting. Int. J. Biochem. Cell Biol. 45, 2200-2208 (2013).

58. Stevens, J. E. et al. Changes in soleus muscle function and fiber morphology with one week of locomotor training in spinal cord contusion injured rats. J. Neurotrauma 23, 1671-1681 (2006).

59. Ohnishi, Y. et al. Disuse muscle atrophy exacerbates motor neuronal degeneration caudal to the site of spinal cord injury. Neuroreport 23, 157-161 (2012).

60. Yoshihara, $\mathrm{H}$. et al. Combining motor training with transplantation of rat bone marrow stromal cells does not improve repair or recovery in rats with thoracic contusion injuries. Brain Res. 1119, 65-75 (2006).

61. Nicola, F. C. et al. Human dental pulp stem cells transplantation combined with treadmill training in rats after traumatic spinal cord injury. Braz. J. Med. Biol. Res. 49, https://doi.org/10.1590/1414-431X20165319 (2016).

62. Kubasak, M. D. et al. OEG implantation and step training enhance hindlimbstepping ability in adult spinal transected rats. Brain 131, 264-276 (2008).

63. Sachdeva, R., Theisen, C. C., Ninan, V., Twiss, J. L. \& Houle, J. D. Exercise dependent increase in axon regeneration into peripheral nerve grafts by propriospinal but not sensory neurons after spinal cord injury is associated with modulation of regeneration-associated genes. Exp. Neurol. 276, 72-82 (2016).

64. Takeoka, A. et al. Axon regeneration can facilitate or suppress hindlimb function after olfactory ensheathing glia transplantation. J. Neurosci. 31, 4298-4310 (2011).

65. Sun, T., Ye, C., Zhang, Z., Wu, J. \& Huang, H. Cotransplantation of olfactory ensheathing cells and Schwann cells combined with treadmill training promotes functional recovery in rats with contused spinal cords. Cell Transplant. 22, S27-S38 (2013).

66. Massoto, T. B. et al. Mesenchymal stem cells and treadmill training enhance function and promote tissue preservation after spinal cord injury. Brain Res. 1726, https://doi.org/10.1016/j.brainres.2019.146494 (2020).

67. Younsi, A. et al. Treadmill training improves survival and differentiation of transplanted neural precursor cells after cervical spinal cord injury. Stem Cell Res. 45, https://doi.org/10.1016/j.scr.2020.101812 (2020).
68. Kadoya, K. et al. Spinal cord reconstitution with homologous neural grafts enables robust corticospinal regeneration. Nat. Med. 22, 479-487 (2016)

69. Ogawa, Y. et al. Transplantation of in vitro-expanded fetal neural progenitor cells results in neurogenesis and functional recovery after spinal cord contusion injury in adult rats. J. Neurosci. Res. 69, 925-933 (2002).

70. Sun, W. M., Ma, C. L., Xu, J. \& He, J. P. Reduction in post-spinal cord injury spasticity by combination of peripheral nerve grafting and acidic fibroblast growth factor infusion in monkeys. J. Int. Med. Res. 49, https://doi.org/10.1177/ 03000605211022294 (2021).

71. Prager, J. et al. Delivery of chondroitinase by canine mucosal olfactory ensheathing cells alongside rehabilitation enhances recovery after spinal cord injury. Exp. Neurol. 340, https://doi.org/10.1016/j.expneurol.2021.113660 (2021).

72. Keyvan-Fouladi, N., Raisman, G. \& Li, Y. Delayed repair of corticospinal tract lesions as an assay for the effectiveness of transplantation of Schwann cells. Glia 51, 306-311 (2005).

73. Kwon, B. K. et al. Demonstrating efficacy in preclinical studies of cellular therapies for spinal cord injury - how much is enough? Exp. Neurol. 248, 30-44 (2013).

74. Dugan, E. A., Jergova, S. \& Sagen, J. Mutually beneficial effects of intensive exercise and GABAergic neural progenitor cell transplants in reducing neuropathic pain and spinal pathology in rats with spinal cord injury. Exp. Neurol. 327, https://doi.org/10.1016/j.expneurol.2020.113208. (2020).

75. Shroff, G. et al. Role of physiotherapy in the mobilization of patients with spinal cord injury undergoing human embryonic stem cells transplantation. Clin. Transl. Med. 5, 41 (2016).

76. Harkema, S. J., Schmidt-Read, M., Lorenz, D. J., Edgerton, V. R. \& Behrman, A. L. Balance and ambulation improvements in individuals with chronic incomplete spinal cord injury using locomotor training-based rehabilitation. Arch. Phys. Med. Rehabil. 93, 1508-1517 (2012).

77. Knikou, M. Plasticity of corticospinal neural control after locomotor training in human spinal cord injury. Neural Plast. 2012, https://doi.org/10.1155/2012/ 254948 (2012).

78. Kapadia, N., Zivanovic, V. \& Popovic, M. R. Restoring voluntary grasping function in individuals with incomplete chronic spinal cord injury: pilot study. Top. Spinal Cord. Inj. Rehabil. 19, 279-287 (2013).

79. Gant, K. L. et al. Body system effects of a multi-modal training program targeting chronic, motor complete thoracic spinal cord injury. J. Neurotrauma 35, 411-423 (2018).

80. Zheng, Y., Mao, Y. R., Yuan, T. F., Xu, D. S. \& Cheng, L. M. Multimodal treatment for spinal cord injury: a sword of neuroregeneration upon neuromodulation. Neural Regen. Res. 15, 1437-1450 (2020).

81. Wagner, F. B. et al. Targeted neurotechnology restores walking in humans with spinal cord injury. Nature 563, 65-71 (2018).

82. Marquez-Chin, C. \& Popovic, M. R. Functional electrical stimulation therapy for restoration of motor function after spinal cord injury and stroke: a review. Biomed. Eng. Online 19, 34 (2020).

83. Nam, K. Y. et al. Robot-assisted gait training (Lokomat) improves walking function and activity in people with spinal cord injury: a systematic review. J. Neuroeng. Rehabil. 14, 24 (2017).

84. Lam, T. et al. A systematic review of the efficacy of gait rehabilitation strategies for spinal cord injury. Top. Spinal Cord. Inj. Rehabil. 13, 32-57 (2007).

85. Seanez-Gonzalez, I. et al. Body-machine interfaces after spinal cord injury: rehabilitation and brain plasticity. Brain Sci. 6, https://doi.org/10.3390/ brainsci6040061 (2016).

86. Samaddar, S. et al. Transspinal direct current stimulation modulates migration and proliferation of adult newly born spinal cells in mice. J. Appl. Physiol. (1985) 122, 339-353 (2017).

87. Rejc, E. \& Angeli, C. A. Spinal cord epidural stimulation for lower limb motor function recovery in individuals with motor complete spinal cord injury. Phys. Med. Rehabil. Clin. N. Am. 30, 337-354 (2019).

88. Harkema, S. et al. Effect of epidural stimulation of the lumbosacral spinal cord on voluntary movement, standing, and assisted stepping after motor complete paraplegia: a case study. Lancet 377, 1938-1947 (2011).

89. Musienko, P., Heutschi, J., Friedli, L., van den Brand, R. \& Courtine, G. Multisystem neurorehabilitative strategies to restore motor functions following severe spinal cord injury. Exp. Neurol. 235, 100-109 (2012).

90. Capogrosso, M. et al. A brain-spine interface alleviating gait deficits after spinal cord injury in primates. Nature 539, 284-288 (2016).

91. Jarocha, D. et al. Preliminary study of autologous bone marrow nucleated cells transplantation in children with spinal cord injury. Stem Cells Transplant. Med. 3 395-404 (2014).

92. Huang, H., Xi, H., Chen, L., Zhang, F. \& Liu, Y. Long-term outcome of olfactory ensheathing cell therapy for patients with complete chronic spinal cord injury. Cell Transplant. 21, S23-S31 (2012) 
93. Oh, S. K. et al. A phase III clinical trial showing limited efficacy of autologous mesenchymal stem cell therapy for spinal cord injury. Neurosurgery 78, 436-447 (2016).

94. Curtis, E. et al. A first-in-human, phase I study of neural stem cell transplantation for chronic spinal cord injury. Cell Stem Cell 22, 941-950.e6 (2018).

95. Levi, A. D. et al. Clinical outcomes from a multi-center study of human neural stem cell transplantation in chronic cervical spinal cord injury. J. Neurotrauma 36, 891-902 (2019).

96. Vaquero, J. et al. Intrathecal administration of autologous mesenchymal stromal cells for spinal cord injury: safety and efficacy of the 100/3 guideline. Cytotherapy 20, 806-819 (2018).

97. Zhu, H. et al. Phase I-II clinical trial assessing safety and efficacy of umbilical cord blood mononuclear cell transplant therapy of chronic complete spinal cord injury. Cell Transpl. 25, 1925-1943 (2016).

98. Tabakow, P. et al. Transplantation of autologous olfactory ensheathing cells in complete human spinal cord injury. Cell Transplant. 22, https://doi.org/10.3727/ 096368912 X663532 (2013).

99. Saberi, H. et al. Treatment of chronic thoracic spinal cord injury patients with autologous Schwann cell transplantation: an interim report on safety considerations and possible outcomes. Neurosci. Lett. 443, 46-50 (2008).

100. Chen, W. et al. NeuroRegen scaffolds combined with autologous bone marrow mononuclear cells for the repair of acute complete spinal cord injury: a 3-year clinical study. Cell Transplant. 29, https://doi.org/10.1177/0963689720950637 (2020).

101. Maher, J. L., Anderson, K. D., Gant, K. L. \& Cowan, R. E. Development and deployment of an at-home strength and conditioning program to support a phase I trial in persons with chronic spinal cord injury. Spinal Cord. 59, 44-54 (2021).

102. Devillard, X., Rimaud, D., Roche, F. \& Calmels, P. Effects of training programs for spinal cord injury. Ann. Readapt. Med. Phys. 50, 490-498 (2007).

103. Wang, L. L. \& Zhang, C. L. Engineering new neurons: in vivo reprogramming in mammalian brain and spinal cord. Cell Tissue Res. 371, 201-212 (2018).

\section{ACKNOWLEDGEMENTS}

This work was supported by The General Insurance Association of Japan, a general research fund for the department of rehabilitation medicine in Kyorin university school of medicine, and by funding from the Japan Agency for Medical Research and Development (AMED) (Grant Number JP20bm0204001, JP19bm0204001, and JP18bk0104017 to H.O. and M.N.).

\section{AUTHOR CONTRIBUTIONS}

Conceptualization, S.T., O.T., O.U., S.Y., and T.T.; Chapter I and conclusion, writingoriginal draft preparation: S.T. and O.T.; writing-review and editing, S.Y., T.T., H.O., and M.N.; Chapter II, writing-original draft preparation: M.S., T.S., T.Y., Y.T., and T.K.; writing-review and editing, M.K., O.U. T.T., and H.O; Chapter III, writing-original draft preparation: S.T., O.T., G.I., Y.K., A.Y., and S.T.; writing-review and editing, K.F. N. N. H.O., and M.N.; Chapter IV, writing-original draft preparation: S.T., M.S., T.S., Y.T., and O.U.; writing-review and editing, M.K., T.T., and M.N.; Chapter V, writingoriginal draft preparation: O.T., M.S., and T.K.; writing — review and editing, H.O.; table preparation and article investigation: T.S., T.Y., Y.T. K.U., T.K., G.I., Y.K. A.Y, S.N., and M.K.; table supervision: K.F. and N.N.; supervision, S.Y., T.T., H.O., and M.N.; funding acquisition, S.T., S.Y., H.O., and M.N. All the authors discussed and commented on the whole article. All authors have read and agreed to the published version of the manuscript.

\section{COMPETING INTERESTS}

M.N. declared a consultancy role with K-Pharma Inc. and research funding from RMic and Hisamitsu. H.O. declared a leadership position at Keio University School of Medicine and is a compensated scientific consultant for San Bio Co. Ltd. and K Pharma Inc. The remaining authors declare no competing interests.

\section{ADDITIONAL INFORMATION}

Supplementary information The online version contains supplementary material available at https://doi.org/10.1038/s41536-021-00191-7.

Correspondence and requests for materials should be addressed to Syoichi Tashiro.

Reprints and permission information is available at http://www.nature.com/ reprints

Publisher's note Springer Nature remains neutral with regard to jurisdictional claims in published maps and institutional affiliations.

(i) Open Access This article is licensed under a Creative Commons adaptation, distribution and reproduction in any medium or format, as long as you give appropriate credit to the original author(s) and the source, provide a link to the Creative Commons license, and indicate if changes were made. The images or other third party material in this article are included in the article's Creative Commons license, unless indicated otherwise in a credit line to the material. If material is not included in the article's Creative Commons license and your intended use is not permitted by statutory regulation or exceeds the permitted use, you will need to obtain permission directly from the copyright holder. To view a copy of this license, visit http://creativecommons. org/licenses/by/4.0/.

(c) The Author(s) 2021 